\title{
Eine Rationenliste aus dem Königreich Arraphe
}

Während auch heute noch der Glanz der altmesopotamischen Reiche oft allein mit den Namen von Königen verbunden wird, ist doch gewiß, daß deren Macht und damit auch die großen Kulturschöpfungen Mesopotamiens ohne die gewaltigen, und sicher nicht immer ganz freiwilligen Leistungen der ,einfachen Arbeiter' nicht hätte entstehen können. Ganz anders als in der klassischen römisch-griechischen Kultur ist diesem Bevölkerungsteil für fast alle historischen Perioden in der kaum überschaubaren Menge von Verwaltungsurkunden ein monumentales Denkmal gesetzt.

Dem Jubilar, Herrn Prof. Brentjes, sei hier eine kleine Urkunde präsentiert, die eine Liste über eine monatliche Ausgabe von Gerstenrationen an Arbeiterinnen und Arbeiter enthält.

Die Urkunde gehörte ursprünglich in die nun aufgelöste Tontafelsammlung des Lord Amherst of Hackney. ${ }^{1}$ Jetzt befindet sie sich in Privatbesitz. Die vermutlich von Th. G. Pinches angebrachte Signatur auf dem für die Amherst-Sammlung typischen halbmondförmigen Etikett lautet N 34. ${ }^{2}$ An der Krümmung der Tafel, die $5,5 \mathrm{~cm}$ breit und nur noch $6 \mathrm{~cm}$ lang ist, ist $\mathrm{zu}$ erkennen, daß nur weniger als die Hälfte des Dokumentes erhalten blieb. Vor allem die Oberfläche der Tafelvorderseite ist sehr stark beschädigt. Auf dem linken Tafelrand sind ein weitgehend vollständiger Siegelabdruck ${ }^{3}$ und Spuren eines weiteren Siegelabdruckes zu erkennen (vgl. das Photo S. 118). Dargestellt sind eine Ziege? (links) und eine Sphin ${ }^{4}$, die einander zugewandt

1 Zu dieser Sammlung und ihrer Geschichte vgl. meine Anmerkung in AoF 17 [1990] $189 \mathrm{ff}$.

${ }^{2}$ Ein solches Etikett ist beispielsweise in Th. G. Pinches, The Amherst Tablets, Part I, London 1908, Pl. IV Nr. 2; auch Pl. V Nr. 4 zu sehen. Ob in der vorliegenden Signatur N 34 N für Nuzi steht, ist unklar.

3 Vergleichbare Siegel hat E. Porada, Seal Impressions of Nuzi, New Haven 1947 (AASOR 24) S. 80 f. in der "Group XXV“ zusammengestellt.

${ }^{4} \mathrm{Zu}$ der liegenden Sphinx mit dem groben, nur durch ein Dreieck angedeuteten Gesicht und der hohen gerundeten Kappe vgl. z. B. Porada, AASOR 24, Nr. 457 und 636; auch B. Parker, Iraq 11 [1949] $14 \mathrm{ff}$. Nr. 34, 75, 77, 90 und 128 (freundlicher Hinweis: Frau Dr. U. Moortgat-Correns; diese Siegel aus Palästina). 
sind. Zwischen den beiden Gestalten sind Spuren zu erkennen, die möglicherweise als Baum ${ }^{5}$ oder als Standarte ${ }^{6}$ zu deuten sind. Über der Szene verlief vielleicht ein Flechtband.

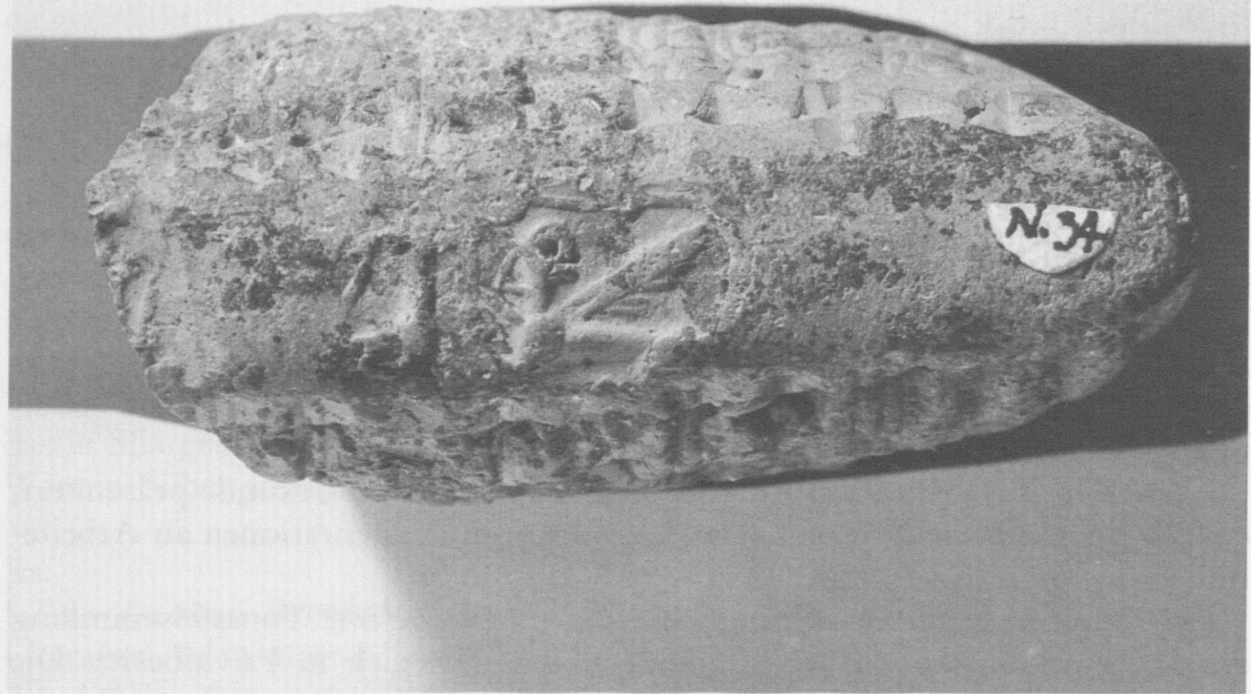

Photo: Margret Nissen

Die Siegelabdrücke, die genannten (weitenteils hurritischen) Personennamen, der Schriftduktus, das Formular der Urkunde und nicht zuletzt die Nennung des Monates šehli in Rs. 13' lassen keinen Zweifel daran, daß die hier vorgestellte Tontafel aus dem Königreich Arraphe, vermutlich aus Nuzi, stammt und wohl im 14. Jh. v. Chr. geschrieben wurde. ${ }^{8}$ Aus der Tafelunterschrift (Rs. 13'f.) wird deutlich, daß in der Tafel Gerstenrationen und -zuteilungen zusamengestellt waren, die im Monat šehli ausgegeben wurden.

5 Vgl. Porada, AASOR 24, Tf. 42/43, S. 80 Gruppe XXV und S. 113.

6 Vgl. Porada, ebd. S. 80, Gruppe XXV mit Nr. 849 ff.

${ }^{7}$ Für diesen Hinweis danke ich Frau Dr. U. Moortgat sehr herzlich. Flechtbänder über Tierszenen finden sich z. B. in: Porada, ebd. Nr. 54, 114, 115, 116, 118, 137, 201 und 743 (Zeichnung Tf. LIII).

8 Zur Datierung der Nuzi-Archive vgl. D. L. Stein, A Reappraisal of the "Sauštatar Letter" from Nuzi, ZA 79 [1989] 36-60. In ZA 76 [1986] 254-288 hat M. P. Maidman weitere unpublizierte Nuzi-Texte, die sich im Britischen Museum befinden, zusammengestellt. 
Ene Rationenliste aus Arraphe

119

Vs.

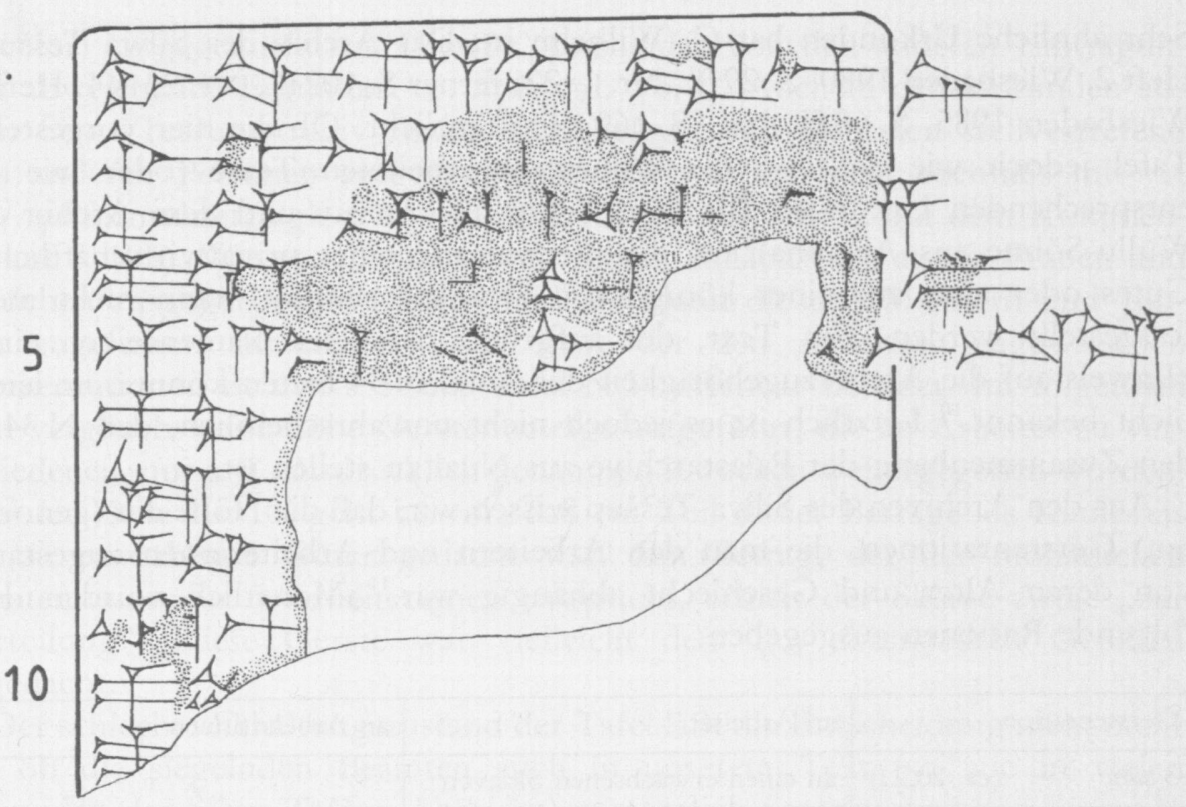

Rs.

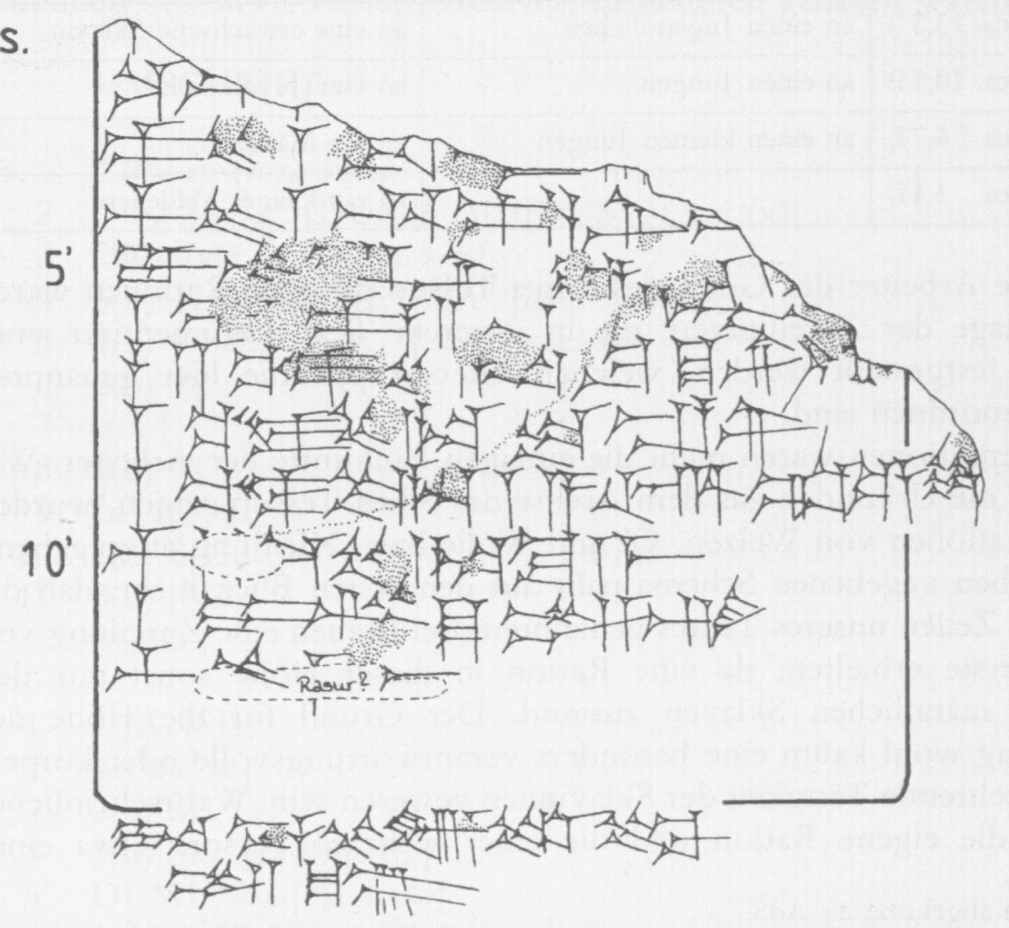

Abb. 1 
Sehr ähnliche Urkunden hat G. Wilhelm in: Das Archiv des Šilwa Teššup?, Heft 2, Wiesbaden 1980, S. 29 ff., Nr. 1-22; ferner S. 102 ff., Nr. 23-44; Heft 3, Wiesbaden 1985, S. 68 ff., Nr. 98-169 veröffentlicht. Ob die hier vorgestellte Tafel jedoch wie die von $G$. Wilhelm bearbeiteten Texte (oder wie die entsprechenden Urkunden aus dem Tehip-tilla-Archiv und dem Archiv der Wullu-Söhne aus Arrapha) aus der Verwaltung eines privatwirtschaftlichen Gutes oder aber aus einer königlichen Domäne stammt, kann nicht mehr festgestellt werden. Ein Text, der aufgrund paralleler Namenreihen einen Hinweis auf die Archivzugehörigkeit der Tafel N 34 bieten könnte, ist leider nicht bekannt. ${ }^{10}$ Letztlich ist es jedoch nicht unwahrscheinlich, daß N 34 in den Zusammenhang der Palastarchive aus Nuzi zu stellen ist.

Aus den Archiven des Šilwa-Teššup wissen wir, daß die Höhe der (genormten) Gerstenrationen, die man den Arbeitern und Arbeiterinnen zugestand, von deren Alter und Geschlecht abhängig war. ${ }^{11}$ Monatlich wurden dort folgende Rationen ausgegeben:

\begin{tabular}{|lr|l|l|}
\hline \multicolumn{2}{|l|}{ Gerstenration } & an Arbeiter & an Arbeiterinnen \\
\hline 3 sütu $\quad$ (ca. 20,2 l) & an einen erwachsenen Sklaven & - \\
\hline 2 sūtu $\quad$ (ca. 13,5l) & an einen Jugendlichen & an eine erwachsene Sklavin \\
\hline 1 sütu $4 q \hat{u}$ & (ca. 10,11) & an einen Jungen & an eine Jugendliche \\
\hline 1 sūtu & (ca. 6,7l) & an einen kleinen Jungen & an ein Mädchen \\
\hline $4 q \hat{u}$ & (ca. 3,41) & - & an ein kleines Mädchen \\
\hline
\end{tabular}

Die für die Arbeiter des Gutes des Šilwa-Teššup gültigen Rationen waren auch Grundlage der Zuteilungen, die in unserem Text dokumentiert sind. Somit kann festgestellt werden, welcher Altersgruppe die hier genannten Personen zuzuordnen sind.

Die Gerstenrationen waren nicht die einzigen Einkünfte der Arbeiter. Wie uns ebenfalls die Urkunden aus dem Archiv des Šilwa-Teššup zeigen, wurden zusätzliche Rationen von Weizen, Öl und Wolle bzw. Kleidung ausgegeben.

$\mathrm{Zu}$ dem oben gegebenen Schema paßt auf den ersten Blick nicht, daß die in den ersten Zeilen unseres Textes genannten drei Frauen eine Zuteilung von je 3 sütu Gerste erhielten, da eine Ration in dieser Höhe sonst nur den erwachsenen männlichen Sklaven zustand. Der Grund für die Höhe der Zuteilung mag wohl kaum eine besonders verantwortungsvolle oder körperlich sehr auszehrende Tätigkeit der Sklavinnen gewesen sein. Wahrscheinlicher ist, daß sie die eigene Ration und die einer weiteren Person (etwa einer

" Im folgenden abgekürzt als Adঙ̆.

1" Für diese Information danke ich Herrn Professor G. Wilhelm herzlich.

$"$ Siehe Wilhelm, Adふ̌ 1, S. 22. Die hier gegebene Tabelle richtet sich nach der Übersicht von Wilhelm, ebd. S. 22. 
Tochter) entgegennahmen. Hierfür spricht das von den anderen Eintragun-

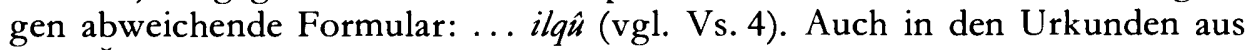
dem Šilwa-Teššup-Archiv finden sich Belege, daß Rationen stellvertretend von anderen Personen entgegengenommen wurden (dort ebenfalls mit ... ilqe /ilqûu formuliert; vgl. z. B. AdŠ 2, S. 107, Nr. 24, 10-12 mit dem Kommentar auf S. 105). Wie es scheint, war es üblich, zunächst die an die Frauen und Mädchen ausgegebenen Rationen und erst dann die den Männern und Jungen zugeteilten aufzulisten. ${ }^{12}$ Die Liste der den Arbeitern zugewiesenen Gerstenrationen ist in Rs. $5^{\prime}$ der Tafel N 34 offenbar beendet. Im folgenden sind vergleichsweise hohe Gerstenbeträge aufgeführt, die an Arbeiter zu verschiedenen, nur $z$. T. ausdrücklich genannten $Z$ wecken weitergegeben wurden. Aus der Zeile Rs. $10^{\prime}$ geht hervor, daß ein Teil dieser Beträge als Kaufpreis für zu erwerbende Güter gedacht war. Ein Betrag, der der monatlichen Gerstenration für 5 Arbeiterinnen entspricht, erhielt der Sklave Artae ,zur Verteilung“. Diese Gerste war vielleicht den ihm unterstellten Gehilfen zugedacht.

Der schlechte Erhaltungszustand der Tafel läßt die Entscheidung nicht mehr $\mathrm{zu}$, ob die siegelnden Beamten auch in unserem Text (so wie in vielen Urkunden des Silwa-Teššup-Archives) namentlich genannt waren.

\section{UMSCHRIFT}

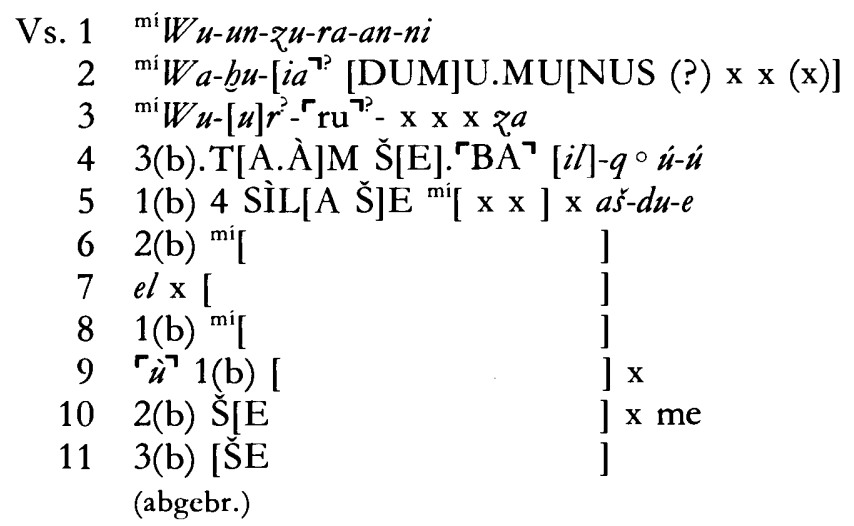

Rs. 1' 1 [ANŠE

$2^{\prime} \quad q a-{ }^{\prime} d u^{\top} \times[$

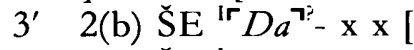

4' 1(b) ŠE 'Hu-un-ni-i[a]

5' DUMU $M u-[r] a-n i-i[a]$

$6^{\prime} \quad 1 \mathrm{AN}[\mathrm{S} E] 2(\mathrm{~b}) 4$ SìlA $a-n a{ }^{\prime} \mathrm{x}-[$

12 Zahlreiche Beispiele hierfür lassen sich in AdŠ finden. 
7' 4 ANŠE ŠE $r a^{\top}-n a \check{s} u-k u-n i$

8' 1 ANŠE ŠE $a-n a t e_{4}-e-n i$

9' 1 ANŠE ŠE $a$-na ' $A$-ar-ta-e SUM

$10^{\prime}$ 1(b) \{Rasur: 2 (oder: 4) SìLA\} ŠE $a-n a$ KUŠ.ME

$11^{\prime} \quad$ 'I-zi-ku il-qè

$12^{\prime} \quad 1$ (b) \{Rasur: ŠE ' $A s^{\prime}$ ]

Leerraum

auf dem unteren Rand:

13' i-na ITI še-eh-li ŠE.BA

$14^{\prime} \quad i l-q u \dot{u}-\dot{u}$

\section{ÜBERSETZUNG}

Vs. 1 (Die Sklavin) Wunzuranni,

2 (die Sklavin) Wahurja], die [To]ch[ter des Tataja ${ }^{3}$,

3 (und die Sklavin Wurru... za

4 haben jeweils 3 sütu (ca. 20,21) Gerste entgegengenommen.

51 sütu 4 qu (ca. 10,1 l) Gerste (für die Jugendliche) ... ašdue;

62 sütu (ca. 13,5l) 〈Gerste〉 (für die Sklavin) [ ];

$7 \quad \ldots$ [ ]

81 sūtu (ca. 6,7 l) (für das Mädchen) [

9 und 1 sütu (ca. 6,7l) [(für das Mädchen/den kleinen Jungen)

102 sütu (ca. 13,5l) Ger[ste für

113 sütu (ca. 20,2l) [Gerste für

(abgebr.)

Rs. 1' 1 [emāru (ca. 67,4l) (+ x) Gerste an/für

$2^{\prime}$ nebst [

3' 2 sütu (ca. 13,5 l) Gerste (für den Jugendlichen) Da... [ ];

4' 1 sütu (ca. 6,7l) Gerste (für den kleinen Jungen) Hunnij[a],

$5^{\prime}$ den Sohn des Mūrānīj][a];

6' 1 emāru 2 sütu 4 qû (ca. 84,3 l) an (den Sklaven)... [ ];

7' 4 emāru (ca. 269,61) Gerste zur Verteilung,

8' 1 emāru $(67,4$ l) Gerste zum Mahlen,

9' 1 emäru $(67,41)$ Gerste \{ohne Zweckangabe\} sind dem Artae gegeben.

$10^{\prime} \quad 1$ sütu (ca. 6,7 l) Gerste (als Kaufpreis) für Lederhäute

$11^{\prime}$ hat Iziku entgegengenommen.

$12^{\prime} \quad$ Eintragung gelöscht

13' Im Monat šehli (Okt./Nov.) hat man die Gerstenrationen

$14^{\prime}$ entgegengenommen. 


\section{KOMMENTAR}

1. Der Frauenname Wunzuranni ist bereits aus HSS 14, 511:34 und aus HSS 16, $381: 30$ bekannt.

2. Eine Arbeiterin Wahuja, Tochter des Ta-ta-a-a, die wie ihr Vater als Sklave im Dienste des Palastes von Turša ${ }^{13}$ stand, ist aus HSS 13, 352:10 bekannt (vgl. auch HSS 13, 429:15f.; Belege: E. Cassin und J.-J. Glassner, Anthroponymie et Anthropologie de Nuzi [AAN], Vol. I, Les Anthroponymes, Malibu 1977, 164a). Möglicherweise ist in der hier bearbeiteten Urkunde eben diese Sklavin genannt. Vielleicht gehörte auch der in Rs. 11' genannte Iziku zu dem Personal des Palastes von Turša (vgl. den Kommentar zu dieser Zeile sowie zu Rs. 9').

3. Das zweite Element des Namens lautete vielleicht $-z_{[i]}[\mathrm{L} i z]-z^{a}$ (hierzu vgl. I. J. Gelb - P. M. Purves - A. A. MacRae, Nuzi Personal Names [NPN],

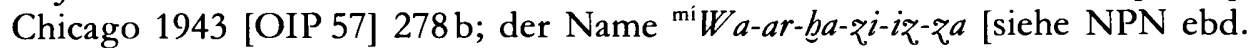
und $172 \mathrm{~b}]$ scheint hier jedoch nicht vorzuliegen). Die Lesung $W u$-ur-rubleibt unsicher. Das Element $W u r r u$ - ist zwar in hurritischen Personennamen aus Nuzi gut bezeugt (siehe NPN 174b und 276a; AAN 168b), aber unter diesen Belegen findet sich bislang kein Frauenname.

5. -aśdue ist wohl das zweite Element des vorliegenden Namens. Der Frauenname Ašdue ist in den Nuzi-Texten belegt (siehe AAN $36 \mathrm{a}$ s.v. 'AŠTUI; weitere Belege für diesen Namen [mit Varianten Aštue, Ašduja etc.] sind in NPN 206 zusammengestellt; vgl. ferner AAN 39b s. v. ${ }^{\mathrm{f}} \mathrm{AZUE}$ ).

9f. Die Zuordnung der auf der Tafelrückseite erhaltenen geringfügigen Spuren zu den Zeilen Vs. 9 und 10 ist nicht ganz gesichert.

4'f. Sowohl der Name Hunnija (NPN 63 a) als auch der akkadische Name Mūrānīja (NPN 99a) sind aus den Nuzi-Texten wohlbekannt. Hunnija, der Sohn des Mūrānīja, ist hier jedoch das erste Mal belegt. Aus der geringen Höhe der Zuteilung darf man wohl schließen, daß Hunnija zum Zeitpunkt der Abfassung der Urkunde noch ein kleiner Junge war.

7'. Zu šukunu, „Verteilung“" vgl. die Ausführungen von G. Wilhelm in AdŠ 2, S. 106.

9'. Belege für den Namen Artae sind in NPN 33a und in AAN 13a zusammengestellt. Einen $A$ - $[a r]$-ta-e, der Sklave des Palastes von Ulamme ${ }^{14}$ war und zu Erntearbeiten in Turša eingesetzt wurde, kennen wir aus HSS 16, 404:28. Falls dieser Artae mit dem in unserem Text genannten identisch sein sollte, könnte man insgesamt drei der in der vorliegenden Urkunde erhaltenen Personennamen für die Vermutung heranziehen, daß die in der Urkunde aus

13 Zu der Stadt Turša, die am südlichen Ufer des unteren Zāb, an der Grenze zum Reich der mittelassyrischen Könige lag, vgl. Fadhil, BaF 6, 193-251.

$14 \mathrm{Zu}$ Ulamme siehe A. Fadhil, BaF 6, 258-276. 
der ehemaligen Amherst-Sammlung genannten Arbeiter, vielleicht auch nur ein Teil von ihnen, aus Turša stammten.

$10^{\prime}$. In den Listen über Gerstenrationen aus dem Archiv des Šilwa-Teššup ist wie in diesem Text - mehrfach eine Ausgabe von Gerste verbucht, die zum Kauf von Leder benötigt wurde [ŠE(.MEŠ) $a$-na KUŠ(.MEŠ); siehe z. B. G. Wilhelm, AdŠ 2, S. 45 Nr. 4:104; S. 115 Nr. 27:12; AsŠ 3, S. 83 Nr. 106, 13; S. 91 Nr. 109:32f. (32: [a-n]a ši-me ' $a-n a\urcorner 2$ KUŠ.ME ša en-₹[i); S. 98 Nr. 112:6 (dort: $a+n a 3$ KUS ša $\left.\mathrm{GU}_{4}\right)$; S. 102 Nr. 113, 34 f.; S. 114 Nr. 118:22; S. 124 Nr. 122:35; S. 133 Nr. 126: 23 und 25; S. 135 Nr. 128:21; S. 148 Nr. $141: 10$ ]. Auch Öl wurde zum Kauf von Leder ausgegeben: AdŠ 3, S. 36 Nr. $72: 12$ und S. 39 Nr. $74: 13$.

14'. Zum Monat šehli vgl. G. Wilhelm, AdŠ 2, S. 28 und AdŠ 3, S. 56. Der Monat šhli entspricht vermutlich dem Monat Oktober/November unseres Kalenders. 